\title{
AJUSTE DE DADOS EXPERIMENTAIS DA SOLUBILIDADE DA UREIA EM MISTURAS DE ISOPROPANOL+ÁGUA
}

\author{
I. A. MAIONCHI ${ }^{1}$, A. G. A. F. MOTTA ${ }^{1}$, A. P. $\operatorname{SILVA}^{1}$ e R. A. MALAGONI ${ }^{1}$ \\ ${ }^{1}$ Universidade Federal de Uberlândia / Faculdade de Engenharia Química \\ E-mail para contato: malagoni@feq.ufu.br
}

\begin{abstract}
RESUMO - A ureia, produzida a partir de amônia e dióxido de carbono, possui $46 \%$ de sua massa molar de nitrogênio, sendo assim, classificada como uma carbamida. Neste trabalho, estudou-se sua solubilidade em misturas de isopropanol + água com porcentagens volumétricas de isopropanol $33,3 \%$ e $66,7 \%$ na temperatura de 278,15 a 333,15 K. O experimento é realizado em temperatura constante com agitação de 2 horas e 2 horas de repouso. Após a coleta dos dados, fez-se os cálculos da solubilidade da ureia, os quais são baseados no método gravimétrico. Em seguida, fez-se o ajuste dos dados experimentais que consiste na definição dos parâmetros da equação escolhida, dos seus respectivos erros e a significância dos mesmos. A partir do ajuste, plotou-se os gráficos da solubilidade em função da temperatura, possibilitando a visualização da solubilidade em temperaturas não obtidas experimentalmente. Os resultados obtidos são condizentes com a literatura.
\end{abstract}

\section{INTRODUÇÃO}

A ureia, um composto orgânico cristalino, de cor branca, inodoro, tóxico, higroscópico altamente solúvel em água, é considerada como um composto nitrogenado não proteico (NNP), sendo classificada quimicamente como amida. Possui massa molar de 60,06 kg/kmol, densidade de 1.330 $\mathrm{kg} / \mathrm{m}^{3}$ e ponto de fusão de 406,15 K (Unido e Ifdc, 1998; Leão et al., 2011; Carvalho et al., 2012).

O método gravimétrico está entre os mais amplamente aplicados de todos os métodos analíticos. Eles têm sido desenvolvidos para a maioria dos cátions e ânions inorgânicos, como também para as espécies neutras como água, dióxido de enxofre, dióxido de carbono e iodo. Uma grande variedade de substâncias orgânicas também pode ser determinada por gravimetria. Alguns exemplos são a lactose em derivados de leite, em preparações farmacêuticas, fenolftaleína em laxantes, nicotinas em pesticidas (Malagoni, 2006). O objetivo deste trabalho foi determinar a solubilidade da ureia em misturas isopropanol + água, com frações de isopropanol de 33,3\% e 66,7\%, com temperaturas variando de 278,15 a $333,15 \mathrm{~K}$. Assim como analisar e tratar os resultados estatisticamente, determinando o desvio absoluto das medidas de solubilidade e fazendo ajustes dos dados experimentais.

\section{MATERIAL E MÉTODOS}


Nos experimentos de determinação dos dados de solubilidade do sistema ureia em isopropanol, foram utilizados: ureia (Nuclear, pureza: 99,9\%), isopropanol (Vetec, pureza: 99,5\%) e água bidestilada e deionizada (Marte, DM-50). A unidade experimental utilizada nesse trabalho é apresentada na Figura 1. Uma célula de vidro borossilicato encamisada foi ligada a um banho termostatizado (Marconi, MA-184), a fim de manter a temperatura da célula constante ou com a mínima variação possível. Foi acoplado no topo da célula, um termopar ligado a um indicador de temperatura (FullGauge, TIC-17RGTi) para monitorar a temperatura da solução. Utilizou-se um agitador magnético (Marconi, MA-089) que através de uma barra magnética $(1,512 \mathrm{~cm}$ de comprimento e 0,607 cm de diâmetro) revestida de teflon promoveu a agitação da mistura, proporcionando uma homogeneização da mistura soluto + solvente.

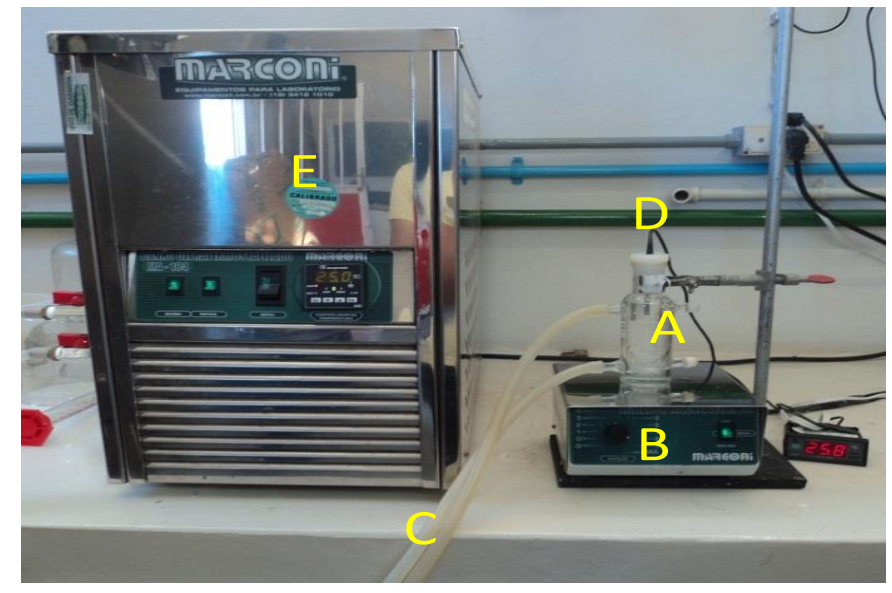

Figura 1 - Unidade experimental: (A) - Célula de equilíbrio; (B) - Agitador magnético; (C) Mangueiras de látex; (D) - Termopar; (E) - Banho Termostatizado.

Foi utilizada neste trabalho uma célula de equilíbrio de vidro, conectada com o banho termostatizado através das mangueiras de látex. Colocou-se na célula uma solução de isopropanol + água na porcentagem volumétrica desejada. Foi inserido na solução, um termopar calibrado e quando a temperatura da solução atingiu o desejado, iniciou-se a agitação da solução que estava com excesso de ureia para que ela ficasse saturada, por 2 horas. Após esse tempo, desligou-se o agitador para que o sistema entrasse em equilíbrio. O período de repouso foi de 2 horas. Retirou-se então, 4 amostras de aproximadamente $3 \mathrm{~mL}$.

Para a determinação da solubilidade da ureia em uma mistura de solventes isopropanol + água, foi utilizada o método gravimétrico. Os béqueres foram pesados quando ainda secos e limpos, logo após, as amostras da célula de equilíbrio foram retiradas, pesadas e levadas para a secagem numa estufa por 96 horas a 333,15K. Após essa secagem, as amostras que continham apenas ureia cristalizada foram pesadas por cerca de 4 dias até massa ficar constante. Através dos dados coletados, foi possível determinar a massa de ureia e a massa do solvente presente em cada amostra, o que permitiu que a solubilidade da ureia fosse calculada.

\section{RESULTADOS E DISCUSSÃO}


A solubilidade da ureia foi calculada para cada temperatura com os dados coletados: massa dos béqueres, massa da amostra com o solvente e massa da amostra totalmente seca. Com a manipulação destes dados e o uso da fórmula abaixo, foram obtidos os dados de solubilidade da ureia para a porcentagem volumétrica de isopropanol de: $33,3 \%$ e $66,7 \%$.

$$
S=\frac{m_{u}}{m_{s}} \times 100
$$

Sendo: $S$ a solubilidade (g/100 g), $m_{u}$ a massa da ureia $(\mathrm{g})$ e $m_{s}$ a massa do solvente $(\mathrm{g})$.

A Tabela 1 possui dados de solubilidade da ureia em 33,3\% de isopropanol e $66,7 \%$ de água (v/v). A Tabela 2 em 66,7\% de isopropanol e 33,3\% de água (v/v).

Tabela 1 - Solubilidade da ureia em 33,3\% de isopropanol

\begin{tabular}{cc}
\hline $\begin{array}{c}\text { Temperatura } \\
(\mathbf{K})\end{array}$ & $\begin{array}{c}\text { Solubilidade } \\
(\mathbf{g} / \mathbf{1 0 0 g} \text { solvente })\end{array}$ \\
\hline $279,5 \pm 0,1$ & $54,967 \pm 0,368$ \\
$289,5 \pm 0,1$ & $60,307 \pm 3,086$ \\
$298,9 \pm 0,6$ & $92,538 \pm 1,633$ \\
$308,8 \pm 0,1$ & $111,321 \pm 1,118$ \\
$319,0 \pm 0,1$ & $151,332 \pm 0,648$ \\
$328,0 \pm 0,1$ & $162,773 \pm 3,269$ \\
$337,1 \pm 0,4$ & $220,899 \pm 8,568$ \\
\hline
\end{tabular}

Tabela 2 - Solubilidade da ureia em $66,7 \%$ de isopropanol

\begin{tabular}{cc}
\hline $\begin{array}{c}\text { Temperatura } \\
(\mathbf{K})\end{array}$ & $\begin{array}{c}\text { Solubilidade } \\
(\mathbf{g} / \mathbf{1 0 0 g} \text { solvente })\end{array}$ \\
\hline $280,2 \pm 0,2$ & $30,462 \pm 1,735$ \\
$289,2 \pm 0,1$ & $31,8798 \pm 3,978$ \\
$298,4 \pm 0,1$ & $47,932 \pm 0,836$ \\
$309,3 \pm 0,1$ & $61,231 \pm 1,251$ \\
$317,2 \pm 0,2$ & $75,371 \pm 0,821$ \\
$327,0 \pm 0,2$ & $96,350 \pm 2,243$ \\
$335,8 \pm 0,2$ & $125,134 \pm 0,869$ \\
\hline
\end{tabular}

\subsection{Ajustes dos dados da solubilidade da ureia}

Fez-se uso de alguns modelos matemáticos, a fim de verificar a possibilidade de correlacionar os dados de solubilidade com a temperatura. Tais modelos foram equações publicadas na literatura. A 
Equação 2 apresenta o modelo proposto por Lee e Lahti (1972), no estudo da solubilidade da ureia em misturas de solventes. A equação empírica, desenvolvida por Yaws et al. (1993), foi utilizada também para correlacionar os dados deste trabalho, Equação 3.

$$
\begin{aligned}
& \ln S=A+B T \\
& \log S=A^{\prime}+\frac{B^{\prime}}{T}+\frac{C^{\prime}}{T^{2}}
\end{aligned}
$$

A Tabela 3 apresenta os valores dos parâmetros das equações que se ajustaram aos pontos experimentais da solubilidade da ureia em uma mistura de 33,3\% isopropanol e 66,7\% de água, seus respectivos desvios e coeficientes de determinação.

Tabela 3 - Parâmetros das equações de Lee e Lahti e Yaws para 33,3\% isopropanol

\begin{tabular}{cccc}
\hline \multicolumn{2}{c}{ Equação de Lee e Lahti } & \multicolumn{2}{c}{ Equação de Yaws } \\
\hline Parâmetros & Desvio & Parâmetros & Desvio \\
$A=-2,7166$ & 0,5319 & $A^{\prime}=7,0162$ & 4,4403 \\
$B=0,0240$ & 0,0016 & $B^{\prime}=-2054,3322$ & 2783,6506 \\
& & $C^{\prime}=160531,0265$ & 4351705785 \\
$R^{2}=0,9823$ & & $R^{2}=0,9827$ & \\
\hline
\end{tabular}

Para um nível de significância de 5\% no software Statistica, para a fração de 33,3\% isopropanol, foi possível verificar que os parâmetros $A$ e $B$ do modelo de Lee e Lahti foram significativos. Já no modelo de Yaws et al., não houve nenhum parâmetro significativo. Assim, o modelo que melhor se ajusta aos dados experimentais é o modelo de Lee e Lahti, que possui todos os parâmetros significativos.

A Tabela 4 apresenta os valores observados, preditos e residuais da Equação de Lee e Lahti (1972). Já a Tabela 5 apresenta estes valores para a correlação de Yaws et al.(1993).

A Figura 2 apresenta os valores experimentais obtidos em laboratório, juntamente com as equações de Lee e Lahti (1972) e Yaws et al. (1993), as quais descrevem o comportamento dos pontos. 
Tabela 4 - Valores observados e preditos e seus respectivos residuais para a Equação de Lee e Lahti (1972) para 33,3\% isopropanol

\begin{tabular}{cccc}
\hline $\boldsymbol{T}$ & $\begin{array}{c}\text { Observado } \\
(\mathbf{K})\end{array}$ & $\begin{array}{c}\text { Predito } \\
\text { (g/100g solvente) }\end{array}$ & $\begin{array}{c}\text { Residual } \\
(\mathbf{g} / \mathbf{1 0 0 g} \text { solvente) }\end{array}$ \\
$(\mathbf{g} / \mathbf{1 0 0 g}$ solvente $)$ \\
\hline $279,5 \pm 0,1$ & 54,9670 & 54,5614 & 0,4056 \\
$289,5 \pm 0,1$ & 60,3070 & 69,3806 & $-9,0736$ \\
$298,9 \pm 0,6$ & 92,538 & 86,9621 & 5,5759 \\
$308,8 \pm 0,1$ & 111,3210 & 110,3163 & 1,0047 \\
$319,0 \pm 0,1$ & 151,3320 & 140,9548 & 10,3772 \\
$328,0 \pm 0,1$ & 162,7730 & 174,9837 & $-12,2107$ \\
$337,1 \pm 0,4$ & 220,8990 & 217,7503 & 3,1487 \\
\hline
\end{tabular}

Tabela 5 - Valores observados e preditos e seus respectivos residuais para a Equação de Yaws et al.(1993) para 33,3\% isopropanol

\begin{tabular}{cccc}
\hline $\boldsymbol{T}$ & $\begin{array}{c}\text { Observado } \\
(\mathbf{K})\end{array}$ & $\begin{array}{c}\text { Predito } \\
\text { (g/100g solvente) }\end{array}$ & $\begin{array}{c}\text { Residual } \\
(\mathbf{g} / \mathbf{1 0 0 g} \text { solvente) }\end{array}$ \\
$(\mathbf{g} / \mathbf{1 0 0 g}$ solvente)
\end{tabular}

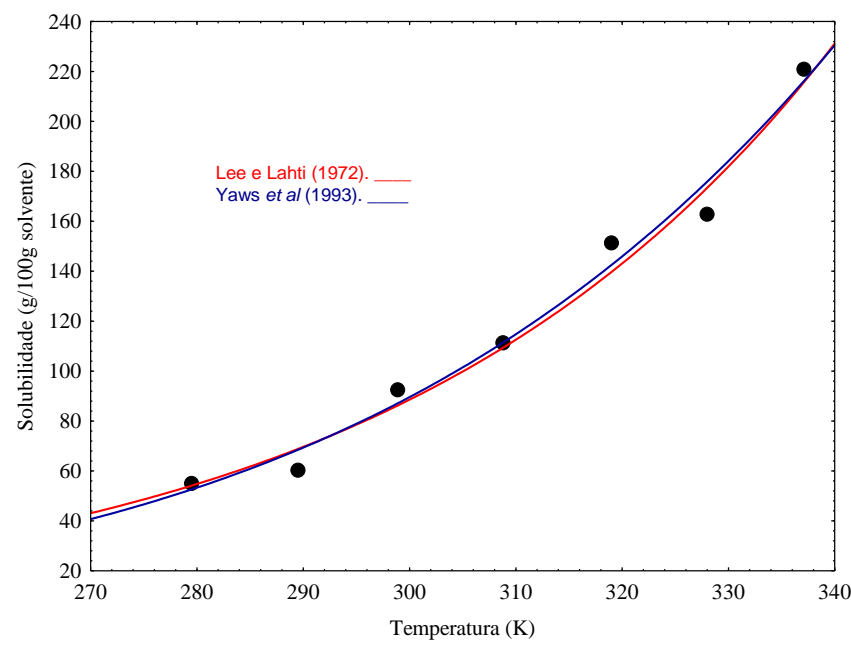

Figura 2 - Gráfico com os pontos experimentais e os ajustes para 33,3\% isopropanol. 
A Tabela 6 apresenta os valores dos parâmetros das equações que se ajustaram aos pontos experimentais da solubilidade da ureia em uma mistura de 66,7\% isopropanol e 33,3\% de água, seus respectivos desvios e coeficientes de determinação.

Tabela 6 - Parâmetros das equações de Lee e Lahti e Yaws para 66,7\% isopropanol

\begin{tabular}{cccc}
\hline \multicolumn{2}{c}{ Equação de Lee e Lahti } & \multicolumn{2}{c}{ Equação de Yaws } \\
\hline Parâmetros & Desvio & Parâmetros & Desvio \\
$A=-4,1606$ & 0,2839 & $A^{\prime}=10,0309$ & 2,3597 \\
$B=0,0268$ & 0,0009 & $B^{\prime}=-3996,8655$ & 1477,8648 \\
& & $C^{\prime}=446393,6558$ & 230832,9406 \\
$R^{2}=0,9959$ & & $R^{2}=0,9961$ \\
\hline
\end{tabular}

Tabela 7 - Valores observados e preditos e seus respectivos residuais para a Equação de Lee e Lahti para $66,7 \%$ isopropanol

\begin{tabular}{|c|c|c|c|}
\hline $\begin{array}{c}T \\
(\mathbf{K})\end{array}$ & $\begin{array}{c}\text { Observado } \\
\text { (g/100g solvente) }\end{array}$ & $\begin{array}{c}\text { Predito } \\
\text { (g/100g solvente) }\end{array}$ & $\begin{array}{c}\text { Residual } \\
\text { (g/100g solvente) }\end{array}$ \\
\hline $280,2 \pm 0,2$ & 30,4620 & 28,0476 & 2,4144 \\
\hline $289,2 \pm 0,1$ & 31,8798 & 35,6813 & $-3,8015$ \\
\hline $298,4 \pm 0,1$ & 47,9320 & 45,6361 & 2,2959 \\
\hline $309,3 \pm 0,1$ & 61,2310 & 61,0835 & 0,1475 \\
\hline $317,2 \pm 0,2$ & 75,3710 & 75,4556 & $-0,0846$ \\
\hline $327,0 \pm 0,2$ & 96,3500 & 98,0685 & $-1,7185$ \\
\hline $335,8 \pm 0,2$ & 125,1340 & 124,0941 & 1,0399 \\
\hline
\end{tabular}

Tabela 8 - Valores observados e preditos e seus respectivos residuais para a Equação de Yaws et al.(1993) para 66,7\% isopropanol

\begin{tabular}{|c|c|c|c|}
\hline $\begin{array}{c}T \\
(\mathbf{K})\end{array}$ & $\begin{array}{c}\text { Observado } \\
\text { (g/100g solvente) }\end{array}$ & $\begin{array}{c}\text { Predito } \\
\text { (g/100g solvente) }\end{array}$ & $\begin{array}{c}\text { Residual } \\
\text { (g/100g solvente) }\end{array}$ \\
\hline $280,2 \pm 0,2$ & 30,4620 & 28,8599 & 1,6021 \\
\hline $289,2 \pm 0,1$ & 31,8798 & 35,9610 & $-4,0813$ \\
\hline $298,4 \pm 0,1$ & 47,9320 & 45,4878 & 2,4442 \\
\hline $309,3 \pm 0,1$ & 61,2310 & 60,6464 & 0,58459 \\
\hline $317,2 \pm 0,2$ & 75,3710 & 75,0084 & 0,3626 \\
\hline $327,0 \pm 0,2$ & 96,3500 & 97,9034 & $-1,5534$ \\
\hline $335,8 \pm 0,2$ & 125,1340 & 124,5012 & 0,6328 \\
\hline
\end{tabular}

A Figura 3 apresenta os valores experimentais obtidos em laboratório, juntamente com as equações de Lee e Lahti (1972) e Yaws et al.(1993), as quais descrevem o comportamento dos pontos. 


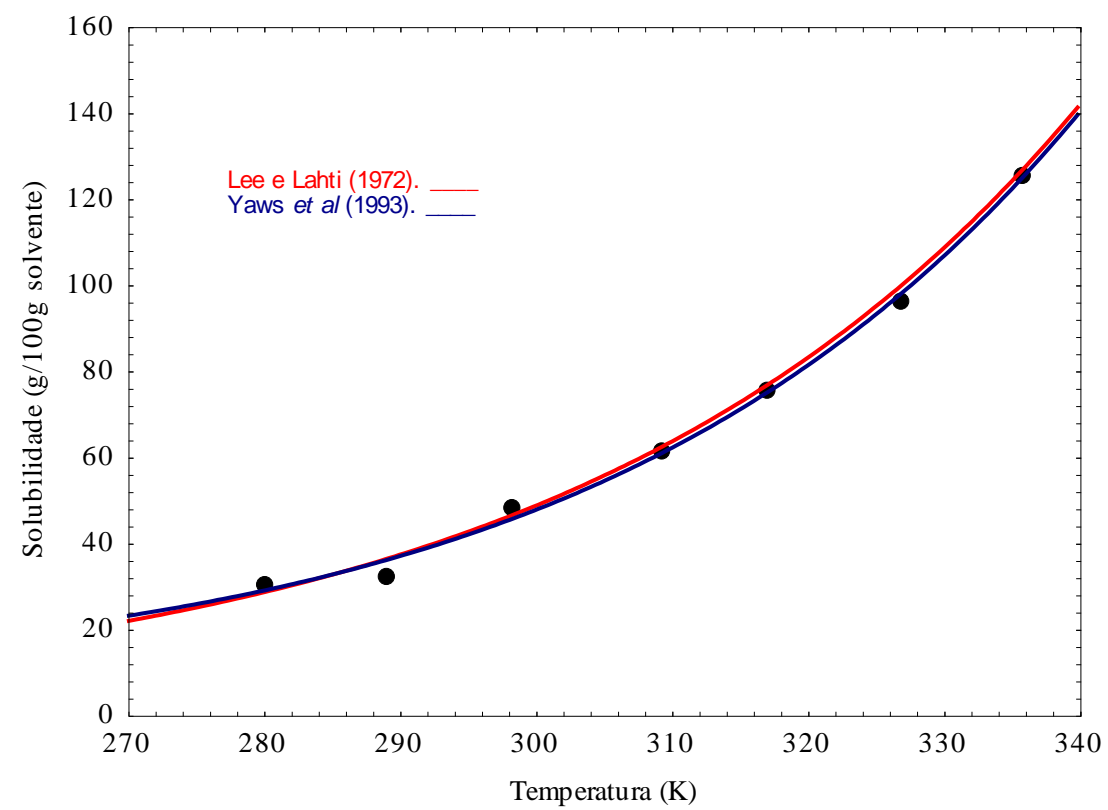

Figura 3 - Gráfico com os pontos experimentais e os ajustes para 66,7\% isopropanol

\section{CONCLUSÃO}

Verificando a influência da temperatura na solubilidade da ureia em isopropanol, pode-se concluir que a solubilidade da ureia aumentou em função do aumento da temperatura. Analisando-se os dados obtidos neste trabalho, verifica-se que os resultados foram coerentes, uma vez que os dados de solubilidade da ureia na fração $66,7 \%$ isopropanol estão abaixo dos valores de solubilidade da ureia na fração de $33,3 \%$ isopropanol, já que a ureia é menos solúvel em isopropanol que em água pura.

O aparato experimental utilizado mostrou-se eficiente na determinação dos dados de solubilidade da ureia em isopropanol por gravimetria, uma vez que os desvios padrão das amostras foram baixos. O modelo de Lee e Lahti (1972) usado para correlacionar os dados mostrou-se bastante significativo nos experimentos realizados com 33,3\% e 66,7\% isopropanol.

\section{NOMENCLATURA}

$A$ - parâmetro da Equação de Lee e Lahti

$B$ - parâmetro da Equação de Lee e Lahti

$A^{\prime}$ - parâmetro da Equação de Yaws et al.

$B^{\prime}$ - parâmetro da Equação de Yaws et al. 
$C^{\prime}$ - parâmetro da Equação de Yaws et al.

$m_{u}$ - massa de ureia em gramas

$m_{s}$ - massa da mistura de solventes em gramas

$S$ - solubilidade (g/ $100 \mathrm{~g}$ de mistura de solventes)

$T$ - temperatura em Kelvin

\section{AGRADECIMENTOS}

Agradecemos a Fundação de Amparo à Pesquisa do Estado de Minas Gerais (FAPEMIG) pelos recursos concedidos no Projeto de Participação Coletiva em Eventos Técnico-Científicos (PCE00082-14) e a Faculdade de Engenharia Química da Universidade Federal de Uberlândia pela estrutura física disponibilizada para a realização desta pesquisa. Agradecemos ao Conselho Nacional de Desenvolvimento Científico e Tecnológico (CNPq) pelo apoio com a bolsa de Iniciação Científica.

\section{REFERÊNCIAS}

CARVALHO, N. D.; CASTRO, C. C.; LOBATO, F. S. ; MALAGONI, R. A. Solubilidade da ureia em etanol+água. In: XIX COBEQ - Congresso Brasileiro de Engenharia Química, 2012, Búzios RJ. Anais do XIX COBEQ, 2012, 5421-5430.

LEÃO, A. M.; CARVAlHO, N. D.; LOBATO, F. S.; MALAGONI, R. A. Determinação experimental da solubilidade de ureia em água. In: VI CBTermo - Congresso Brasileiro de Termodinâmica Aplicada, 2011, Salvador - BA. Anais do VI CBTermo, 2011, 1-6.

LEE, F-M.; LAHTI, L. E. Solubility of urea in water-alcohol mixtures. J. Chem. and Eng. Data, 17, 3 (1972), 304-306.

MALAGONI, R. A. Determinação experimental da solubilidade de orgânicos em água. 2006. 102 p. Dissertação de Mestrado, Faculdade de Engenharia Química, Universidade Federal de Uberlândia, Uberlândia, 2006.

UNIDO (United Nations Industrial Development Organization; IFDC (International Fertilizer Development Center). Fertilizer Manual. $1^{\text {st }}$ ed. Kluwer Academic Publishers, 1998.615 p.

YAWS, C. L.; Lin, X.; Bu, L. The water solubility of naphtenes. Chem. Eng., v. 10, 122 $-123,(1993)$. 